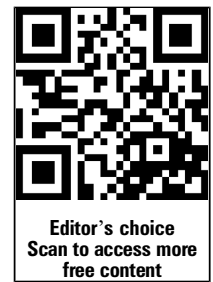

${ }^{1}$ Department of Neurology,
Medical College of Wisconsin and Froedtert Hospital, Milwaukee, Wisconsin, USA 2Department of Neurosurgery, Medical College of Wisconsin and Froedtert Hospital, Milwaukee, Wisconsin, USA ${ }^{3}$ Department of Radiology, Medical College of Wisconsin and Froedtert Hospital, Milwaukee, Wisconsin, USA

Correspondence to Dr Osama 0 Zaidat, Vascular and Interventional Neurology/Department of Neurology, Medical College of Wisconsin/Froedtert Hospital, 9200 West Wisconsin Avenue, Milwaukee, WI 53226, USA; szaidat@mcw.edu

Received 23 May 2013 Revised 21 July 2013 Accepted 24 July 2013 Published Online First 16 August 2013

\title{
Safety and predictors of aneurysm retreatment for remnant intracranial aneurysm after initial endovascular embolization
}

\author{
Mohamed S Teleb, ${ }^{1}$ Dhruvil J Pandya, ${ }^{1}$ Alicia C Castonguay, ${ }^{1}$ Gerald Eckardt, ${ }^{2}$ \\ Rochelle Sweis, ${ }^{1}$ Marc A Lazzaro, ${ }^{1}$ Mohammed A Issa, ${ }^{1}$ Brian-Fred Fitzsimmons, ${ }^{1,2,3}$ \\ John R Lynch, 1,2,3 Osama O Zaidat ${ }^{1,2,3}$
}

\begin{abstract}
Introduction Aneurysmal subarachnoid hemorrhage $(\mathrm{SAH})$ is a rare but devastating form of stroke.

Endovascular therapy has been criticized for its higher rate of recanalization and retreatment. The safety and predictors of retreatment are unknown. We report the clinical outcomes, imaging outcomes and predictors for aneurysm retreatment after initial endovascular embolization.
\end{abstract}

Method We identified patients who underwent endovascular retreatment from July 2005 through November 2011. Aneurysm and patient data were collected. Periprocedural complications were reported as intraoperative perforation (IOP) or thromboembolic event (TEE). Aneurysm and patient characteristics were compared between aneurysms requiring retreatment and those not requiring retreatment to evaluate aneurysm retreatment predictors.

Results A total of 111/871 (13\%) aneurysms underwent retreatment. Two $(0.2 \%)$ were retreated for recurrent acute $\mathrm{SAH}, 82(74 \%)$ aneurysms were located in the anterior circulation, $47(42 \%)$ required stent and $5(5 \%)$ required balloon assist during retreatment. There were a total of 5 (5\%) IOP and 6 (5\%) TEE from which $2(2 \%)$ and $1(1 \%)$ were symptomatic, respectively. Overall symptomatic events rate were $2.7 \%$. Patients were followed up for an average of $15 \pm 14$ months. Seven $(0.8 \%)$ aneurysms required a second retreatment without any recurrent $\mathrm{SAH}$.

Multivariable analysis revealed an OR for aneurysms requiring retreatment of 2.965 for aneurysms presenting as aneurysmal SAH, 1.791 for aneurysms in the posterior circulation and 1.053 for aneurysms with large dome size. Conclusions Aneurysm retreatment is a safe option without a significant increase in morbidity or mortality. $\mathrm{SAH}$, posterior circulation aneurysms and larger aneurysm dome size are predictors of aneurysms requiring retreatment.

\section{INTRODUCTION}

Although aneurysmal subarachnoid hemorrhage (SAH) is a rare form of stroke, it is associated with a high morbidity and mortality and affects younger patients. Owing to this high morbidity and mortality, aneurysms that are unruptured are also routinely treated. The estimated prevalence of cerebral aneurysm ranges from $0.2 \%$ to $9.9 \%$ in the general population and from $10 \%$ to $13.9 \%$ in those with a history of familial aneurysmal SAH. ${ }^{1-4}$ Mortality may be as high as $30-60 \%$ with aneurysm-related SAH. ${ }^{5}$ Currently, surgical clipping and endovascular coiling are the two therapeutic approaches used to treat cerebral aneurysms. The International Subarachnoid Aneurysm Trial (ISAT) as well as the Barrow Ruptured Aneurysm Trial demonstrated that patients with SAH secondary to a ruptured aneurysm who were amenable to treatment with either surgical clipping or endovascular coiling had a better outcome with endovascular therapy at 12 months and during follow-up extended to 7 years. ${ }^{1-2} 6$ However, there is concern about endovascular coiling owing to a higher incidence of aneurysm recurrence with a potential increased risk of recurrent hemorrhage as well as a potential increased procedural risk with need for retreatment. ${ }^{1}{ }^{3-5}$ 7-11 Aneurysm recurrence has been defined as residual contrast filling ${ }^{8}$ and its reported incidence rate is $2-40 \%$ with a rebleeding rate of $0-2 \%$ in ISAT and other larger case series. ${ }^{2} 8912-18$ In aneurysms that have recanalized, additional endovascular treatment can be considered to prevent future rupture, but its safety and predictors for recanalization are unknown at this time. In this study we assessed the safety of retreatment of recurrent aneurysms by evaluating the periprocedural and clinical outcomes and predictors for aneurysms requiring retreatment.

\section{METHODS}

Data were retrospectively collected from July 2005 through November 2011 for consecutive patients who underwent endovascular aneurysm embolization (EAE) at our institution. Patients were included in the study if they underwent retreatment with coil embolization after a primary coil embolization. Recurrence was defined as any aneurysm that had residual neck with demonstrated increase of neck filling over time, regrowth, coil compaction or rebleeding. Most patients had recurrence of aneurysm with aneurysm filling according to the Raymond criteria. ${ }^{19}$ Patients were excluded if they had clipping as their primary treatment modality or if aneurysm retreatment was performed as a planned staging procedure. The need for retreatment was left up to the discretion of the attending physician but, in general, only patients with increased aneurysm neck filling or aneurysm filling underwent retreatment. 
Data extracted were patients' baseline characteristics, primary treatment indications, target aneurysm characteristics (aneurysm location, maximum aneurysm size, neck size), use of assist devices (stent, balloon), clinical outcomes (length of hospital stay, modified Rankin Scale (mRS) score at discharge, discharge disposition), procedural complications (intraoperative perforation (IOP), thromboembolic events (TEE)) and follow-up imaging to assess recurrence either by CT angiography (although only used for one patient with pacemaker after two digital subtraction angiographies (DSAs)), contrast-enhanced MR angiography or DSA. All patients had initial follow-up with DSA at 3 months for ruptured aneurysms and at 6 months for unruptured aneurysms.

The time interval to retreatment was measured in months between the date of primary endovascular coil embolization and date of retreatment. An aneurysm was defined as an anterior cerebral circulation aneurysm if it was located in the internal carotid artery (ICA), anterior cerebral artery, middle cerebral artery, anterior communicating artery (Acom) or posterior communicating artery (Pcom) and as a posterior cerebral circulation aneurysm if it was located in the posterior cerebral artery, superior cerebellar artery, basilar artery, anterior inferior cerebellar artery, posterior inferior cerebellar artery or vertebral artery. Unlike ISAT and other studies, we chose to place Pcom aneurysms in the anterior circulation because of flow dynamics and difference in endovascular approach. ${ }^{2}$ Most Pcom aneurysms are treated via the ICA, as are anterior circulation aneurysms. The maximum diameter and neck size of the aneurysm were measured in millimeters from the DSA. Procedural complications were reported as IOP and TEE and were considered symptomatic if the patient developed a new neurological deficit lasting $\geq 24 \mathrm{~h}$ with an increase in NIH Stroke Scale score of $\geq 2$ compared with baseline.

Predictors for aneurysm retreatment were assessed by comparing aneurysm and patient characteristics during primary treatment in groups 1 (aneurysms requiring retreatment) and 2 (aneurysms not requiring retreatment).

All the procedures were performed in a biplane angiography suite. Patients undergoing stent-assisted coil embolization were premedicated with aspirin 81-325 $\mathrm{mg}$ and clopidogrel $75 \mathrm{mg}$ for 3-7 days prior to the procedure. All procedures were performed under general anesthesia to minimize movement and to have better control of hemodynamics during the procedure. Intravenous heparin was used for systemic anticoagulation, with an activated clotting time (ACT) goal of 250-300 s and frequent ACT checks during the procedures. Patients who presented with SAH did not receive systemic anticoagulation before the procedures; however, systemic anticoagulation was started after the first coil was deployed in the aneurysm with an ACT goal of 200$250 \mathrm{~s}$. After all procedures, patients were admitted to the neurointensive care unit for observation and followed by both the neuroendovascular surgical team and a dedicated neurointensivist.

As per protocol, all patients with a ruptured aneurysm were followed up at 5 weeks, 3 months, 1 year and every year up to 5 years in the clinic. Diagnostic imaging was performed every 3 months for the first year, then at 1 year, and finally noninvasive imaging was performed every year thereafter. Patients with unruptured aneurysms were followed up by conventional angiography at 6 months, then by non-invasive imaging every year for up to 5 years.

\section{Statistical analysis}

Differences between the two groups were tested by univariable analysis using a two-tailed sample $t$ test and $\chi^{2}$ test. Multivariable analysis using a logistic regression model was used to derive ORs of aneurysm retreatment. The multivariable model was adjusted for age, gender, ethnicity, history of hypertension, history of smoking, family history of aneurysms, reason for initial treatment, aneurysm location, stent-assisted initial treatment, balloon-assisted initial treatment, maximum aneurysm dome size, aneurysm neck size and whether the patient required glycoprotein IIB/IIIA inhibitors during the initial treatment. A two-tailed $\mathrm{p}$ value of $<0.05$ was considered to be statistically significant.

\section{RESULTS}

In all, 651 consecutive patients underwent primary EAE for 871 aneurysms from July 2005 through December 2011. A total of 122 aneurysms (14\%) in 100 patients were retreated; two patients had three aneurysms and 18 patients had two aneurysms. Eleven patients (1\%) were excluded, seven because treatment was performed as a staging procedure and four had surgical clipping as their primary treatment, giving a total of $111(13 \%)$ retreated aneurysms after initial EAE included in the analysis (figure 1). None of the aneurysms were pseudoaneurysms. Ten aneurysms had increased neck filling and 112 had aneurysm filling classified as Raymond class 3 .

The mean age of the patients undergoing retreatment was $53 \pm 13$ years, $65(65 \%)$ were women and $82(82 \%)$ were

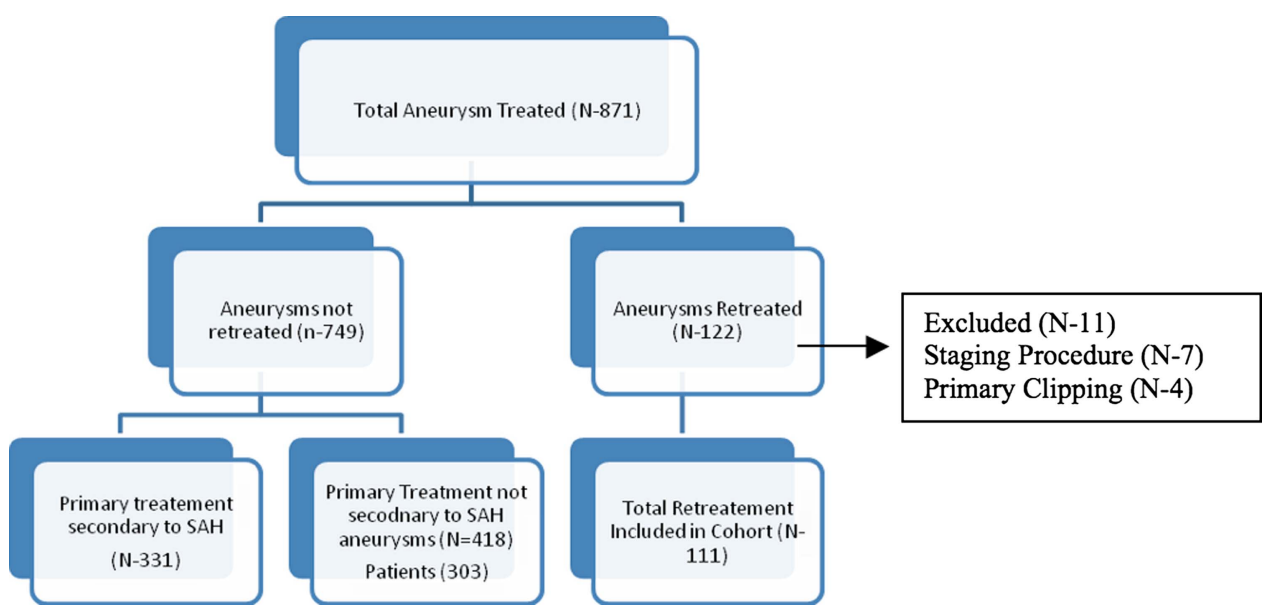

Figure 1 Categorization of aneurysms retreated and aneurysms not retreated in study population. SAH, subarachnoid hemorrhage. 
Caucasians. The mean interval between retreatment after primary treatment was $8 \pm 19$ months. In two patients $(0.2 \%)$, retreatment was performed for an acute SAH. All other cases were retreated after angiographic demonstration of aneurysm recanalization (table 1). Eighty-two aneurysms (74\%) were located in the anterior circulation, $47(42 \%)$ required stents and $5(5 \%)$ required balloon-assisted embolization for retreatment. In the aneurysms undergoing retreatment, the maximum dome size was $7 \pm 4.6 \mathrm{~mm}$ and neck size was $4.4 \pm 3.4 \mathrm{~mm}$ (table 2 ).

There were 11 complications (overall complication rate 10\%, and $2.7 \%$ symptomatic). Of these, five $(5 \%)$ were IOP and six $(5 \%)$ were TEE, of which two $(2 \%)$ and one $(1 \%)$ were symptomatic, respectively, giving a total of three $(2.7 \%)$ symptomatic periprocedural complications. Ninety-three patients (84\%) had a mean length of hospital stay of $\leq 2$ days and 99 patients $(89 \%)$ were discharged home after retreatment. At discharge, 93 patients $(84 \%)$ had a mRS score $\leq 2$ and there were no deaths. Mean follow-up after retreatment was $15 \pm 14$ months; seven patients $(0.8 \%)$ required a second retreatment for recanalization with no re-ruptures. In all seven patients the same aneurysm was retreated (table 3 ).

On univariable analysis, the following variables were statistically significant for aneurysms requiring retreatment: presentation as SAH, posterior circulation aneurysms, stent-assisted primary EAE and larger aneurysm dome size (table 4).

On multivariable analysis, presentation as SAH, posterior circulation and larger aneurysms were significant. The ORs for these multivariable significant variables were 2.965 (95\% CI 1.869 to 4.704$)$ for aneurysms presenting as $\mathrm{SAH}, 1.791$ (95\% CI 1.083 to 2.963$)$ for posterior circulation aneurysms and 1.053 (95\% CI 1.011 to 1.097 ) for larger aneurysm dome sizes. No differences were found in other variables studied for aneurysms requiring retreatment (table 5).

\section{DISCUSSION}

In this study we report our data on the safety and predictors of endovascular aneurysm retreatment. The retreatment rate after primary EAE was $13 \%$. Previously reported rates of aneurysm recurrence have ranged from $15 \%$ to $33 \%,{ }^{4} 161920$ although the retreatment rate has been lower at 8-17\%. ${ }^{21-23}$ The lower rate of retreatment may be secondary to technical challenges to retreatment of an aneurysm due to coil mass compaction and/or the presence of a stent. This lower rate can also be due to reports that fewer than $50 \%$ of recurrences are of sufficient concern to warrant retreatment ${ }^{19} 20$ and that a proportion of aneurysm remnants are stable and unlikely to enlarge or rebleed. ${ }^{8}$ The incidence of rebleeding after primary EAE has been reported to be about $0-2 \% .^{2} 891618$ This rebleeding rate is slightly higher than our rate of $0.2 \%$, which may be because of our aggressive follow-up protocol and lower threshold for retreatment when recanalization occurs.

Table 1 Baseline characteristics of patients undergoing aneurysm retreatment

\begin{tabular}{ll}
\hline Parameter & $\mathrm{N}=100(\%)$ \\
\hline Age (years) & $53 \pm 13$ \\
Women & $65(65 \%)$ \\
White & $82(82 \%)$ \\
Hypertension & $64(64 \%)$ \\
Smoking & $69(69 \%)$ \\
Family history of aneurysms & $15(15 \%)$ \\
\hline
\end{tabular}

Table 2 Characteristics of retreated aneurysms

\begin{tabular}{ll}
\hline Parameter & $\mathrm{N}=111(\%)$ \\
\hline Time interval (months) & $8 \pm 10$ \\
Retreatment secondary to SAH & $2(2 \%)$ \\
Aneurysm in anterior circulation & $82(74 \%)$ \\
Stent-assisted aneurysm retreatment & $47(42 \%)$ \\
Balloon-assisted aneurysm retreatment & $5(5 \%)$ \\
Maximum aneurysm size (mm) & $7 \pm 4.6$ \\
Aneurysm neck size (mm) & $4.4 \pm 3.4$ \\
Aneurysm in anterior circulation & $104(67 \%)$ \\
\hline SAH, subarachnoid hemorrhage. &
\end{tabular}

The natural history of coiled aneurysms is not yet well established, making it very difficult to predict the risk of rebleeding from recanalization. With the high death rate associated with aneurysm rupture, $30-60 \%$ in some studies, ${ }^{5}$ recanalization is of great concern because a treatment protocol has not yet been established in this subgroup of patients. ${ }^{24}$ Once the decision is made to retreat these aneurysms, the periprocedural safety and outcomes are limited to a few studies. Our data support the view that retreatment does not cause a significant increase in morbidity, periprocedural complications or mortality and that only a small number of aneurysms require a second retreatment without any additional risk of rebleeding.

Appropriate follow-up after EAE is another factor that needs to be considered. ${ }^{11}{ }^{19}$ For many clinicians, the challenge to identify which patients are at highest risk and need closer monitoring remains unclear. Knowing the predictors for recanalization or retreatment may help to identify patients needing aggressive versus conservative follow-up. In our study the predictors for retreatment were aneurysms presenting as SAH, those posteriorly located and aneurysms of larger size. In our cohort we demonstrated that one unit of increase in aneurysm dome size increases the risk of retreatment by 5\%. Similar factors influencing aneurysm recurrence have been reported in other studies, including a suboptimal initial angiographic result, packing density ratio, treatment after rupture and aneurysm size. $^{11} 192526$ Unfortunately, we do not have information on packing density, which might have been a factor. More recent studies by Ries et $a l^{21}$ and Renowden et al ${ }^{22}$ show similar results. Although Ries et al only had 33 patients with a retreatment rate of $10 \%$, they classified the different aneurysm sizes and anatomy and concluded that the best predictors of aneurysm retreatment were aneurysm anatomy and the presence of a residual aneurysm after initial treatment. Interestingly, they reported a high rebleeding rate of 15\% (5/33). Renowden et al had a similar total number to our study with a retreatment rate of $8 \%$ (99 patients). They did not compare the retreated patients with the total number of treated patients, so no risk factors were given; however, they reported a similar major complication rate of about 3\%. A systematic review and analysis by Fern et al of 46 studies (8161 patients) found similar risk factors for retreatment with an increased rate of retreatment for posterior circulation aneurysms (defined as vertebrobasilar aneurysms) of $14.5 \%$ compared with $6.5 \%$ for anterior circulation aneurysms as well as a size $>10 \mathrm{~mm}$ using scatterplot regression analysis. ${ }^{27}$

Patients in the ISAT study had a retreatment rate of $17 \%$, with half being early and half being late. ${ }^{23}$ Risk factors for late retreatment were age, larger size and incomplete occlusion on initial treatment. They also had a rebleed rate of $0.6 \%$. Thus, 
Table 3 Aneurysm retreatment procedure and clinical outcomes

\begin{tabular}{ll}
\hline Parameter & $\mathbf{N}=111(\%)$ \\
\hline Intraoperative perforation & $5(5 \%)$ \\
Symptomatic intraoperative perforation & $2(2 \%)$ \\
Thromboembolic events & $6(5 \%)$ \\
Symptomatic thromboembolic events & $1(1 \%)$ \\
Mean length of hospital stay $\leq 2$ days & $93(84 \%)$ \\
Discharged home & $99(89 \%)$ \\
Discharged mRS $\leq 2$ & $93(84 \%)$ \\
Mortality at discharge & 0 \\
Average follow-up (months) & $15 \pm 14$ \\
Recurrent SAH & 0 \\
Aneurysms requiring second retreatment & $7(0.8 \%)$ \\
\hline mRS, modified Rankin Scale; SAH, subarachnoid hemorrhage.
\end{tabular}

overall, the risk factors, percentage of retreatment, rebleed rate and complication rates found in our study are in line with previously published data. The higher rate of retreatment in posteriorly located aneurysms found in our study and in others may be because of the higher rate of recanalization in the vertebrobasilar system compared with the anterior circulation. ${ }^{9}$ Finally, we believe it is important to document these findings in order to inform patients more robustly of their risks and benefits. The other studies were conducted in an era and at centers where many patients underwent clipping. Our data include a more recent population treated predominantly by coiling, which makes it an important contribution for current practice. The limitations of our study include the lack of information on initial packing density and initial occlusion score, the length of follow-up and its retrospective nature.

\section{CONCLUSION}

This study found a $13 \%$ rate of retreatment after primary EAE without a significant increase in morbidity, mortality, periprocedural complications or rebleeding rate after retreatment.

Table 4 Univariable analysis of aneurysms requiring retreatment (group 1) and those not requiring retreatment (group 2)

\begin{tabular}{|c|c|c|c|}
\hline Parameter & $\begin{array}{l}\text { Group } 1 \\
\text { Patients } \mathrm{N}=87 \\
\text { Aneurysms } \\
\mathrm{N}=99\end{array}$ & $\begin{array}{l}\text { Group } 2 \\
\text { Patients } \mathrm{N}=638 \\
\text { Aneurysms } \\
\mathrm{N}=749\end{array}$ & $p$ Value \\
\hline Age, mean $\pm S D$ & $52 \pm 14$ & $55 \pm 14$ & 0.0656 \\
\hline Women & $58(65 \%)$ & $450(71 \%)$ & 0.4564 \\
\hline White & $65(75 \%)$ & $459(72 \%)$ & 0.2476 \\
\hline HTN & $58(67 \%)$ & $371(58 \%)$ & 0.1328 \\
\hline Smoking & $59(69 \%)$ & $407(64 \%)$ & 0.4038 \\
\hline $\begin{array}{l}\text { Family history of } \\
\text { aneurysms }\end{array}$ & $13(15 \%)$ & $87(14 \%)$ & 0.7406 \\
\hline Primary treatment for $\mathrm{SAH}$ & $67(68 \%)$ & $334(45 \%)$ & $1.579 \mathrm{E}-05$ \\
\hline Stent assist & $23(23 \%)$ & $261(35 \%)$ & 0.0232 \\
\hline Balloon assist & $11(11 \%)$ & $84(11 \%)$ & 1.00 \\
\hline Posterior circulation & $25(25 \%)$ & $128(17 \%)$ & 0.0520 \\
\hline Glycoprotein IIB/IIIA use & $12(12 \%)$ & $67(9 \%)$ & 0.3554 \\
\hline Maximum aneurysm size & $8.28 \pm 3.91$ & $7.45 \pm 4.83$ & 0.05 \\
\hline Maximum neck size & $4.14 \pm 2.28$ & $4.06 \pm 3.43$ & 0.7710 \\
\hline
\end{tabular}

$\mathrm{SAH}$, subarachnoid hemorrhage.

Bold type indicates statistically different variables between the two groups.
Table 5 Multivariable analysis probability model for aneurysms requiring retreatment: Wald $\mathrm{Cl}$ for ORs

\begin{tabular}{lll}
\hline Effect & Estimate & $95 \% \mathrm{Cl}$ \\
\hline Reason: SAH vs other & 2.965 & 1.869 to 4.704 \\
Site: posterior vs anterior & 1.791 & 1.083 to 2.963 \\
Maximum dome size & 1.053 & 1.011 to 1.097 \\
\hline SAH, subarachnoid hemorrhage. & &
\end{tabular}

Aneurysms presenting as $\mathrm{SAH}$, posteriorly located aneurysms and those of larger size are likely to increase the risk of retreatment after primary EAE.

Contributors $\mathrm{OOZ}$ and MST were primarily responsible for study design, statistical analysis and writing the manuscript and are the guarantors of the work. DJP, RS, ACC and MAI participated in study design, data collection and composition of the manuscript. MAL, JRL and BFF were involved in data collection and editing of the manuscript.

\section{Competing interests None.}

Ethics approval Ethics approval was obtained from the hospital IRB.

Provenance and peer review Not commissioned; externally peer reviewed.

Data sharing statement A technical appendix, statistical code and dataset are available from the corresponding author at Medical College of Wisconsin. Any transmission of this aneurysm database must be approved by the Medical College of Wisconsin and Froedtert Hospital.

\section{REFERENCES}

1 Molyneux A, Kerr R, Stratton I, et al. International Subarachnoid Aneurysm Trial (ISAT) of neurosurgical clipping versus endovascular coiling in 2143 patients with ruptured intracranial aneurysms: a randomised trial. Lancet 2002;360:1267-74

2 Molyneux AJ, Kerr RS, Yu LM, et al. International Subarachnoid Aneurysm Trial (ISAT) of neurosurgical clipping versus endovascular coiling in 2143 patients with ruptured intracranial aneurysms: a randomised comparison of effects on survival, dependency, seizures, rebleeding, subgroups, and aneurysm occlusion. Lancet 2005;366:809-17.

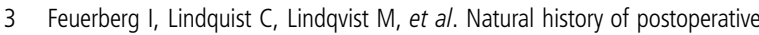
aneurysm rests. J Neurosurg 1987;66:30-4.

4 Thornton J, Debrun GM, Aletich VA, et al. Follow-up angiography of intracrania aneurysms treated with endovascular placement of Guglielmi detachable coils. Neurosurg 2002;50:239-49; discussion 249-50.

5 Asgari S, Wanke I, Schoch B, et al. Recurrent hemorrhage after initially complete occlusion of intracranial aneurysms. Neurosurg Rev 2003;26:269-74.

6 Mcdougal CG, Spetzler RF, Zabramski JM, et al. The Barrow ruptured aneurysm trial. J Neurosurg 2012;161:135-44.

7 Sluzewski M, van Rooij WJ, Slob MJ, et al. Relation between aneurysm volume, packing, and compaction in 145 cerebral aneurysms treated with coils. Radiology 2004;231:653-8.

8 Byrne JV, Sohn MJ, Molyneux AJ, et al. Five-year experience in using coil embolization for ruptured intracranial aneurysms: outcomes and incidence of late rebleeding. J Neurosurg 1999;90:656-63.

9 Cognard C, Weill A, Castaings L, et al. Intracranial berry aneurysms: angiographic and clinical results after endovascular treatment. Radiology 1998:206:499-510.

10 Hope JK, Byrne JV, Molyneux AJ. Factors influencing successful angiographic occlusion of aneurysms treated by coil embolization. AJNR Am J Neuroradiol 1999:20:391-9

11 Cognard C, Weill A, Spelle L, et al. Long-term angiographic follow-up of 169 intracranial berry aneurysms occluded with detachable coils. Radiology 1999;212:348-56.

12 David CA, Vishteh AG, Spetzler RF, et al. Late angiographic follow-up review of surgically treated aneurysms. J Neurosurg 1999;91:396-401.

13 Raymond J, Roy D, Bojanowski M, et al. Endovascular treatment of acutely ruptured and unruptured aneurysms of the basilar bifurcation. J Neurosurg 1997:86:211-19.

14 Kuether TA, Nesbit GM, Barnwell SL. Clinical and angiographic outcomes, with treatment data, for patients with cerebral aneurysms treated with Guglielmi detachable coils: a single-center experience. Neurosurgery 1998;43:1016-25.

15 Manabe H, Fujita S, Hatayama T, et al. Rerupture of coil-embolized aneurysm during long-term observation. Case report. J Neurosurg 1998;88:1096-8. 
16 Murayama Y, Nien YL, Duckwiler G, et al. Guglielmi detachable coil embolization of cerebral aneurysms: 11 years' experience. J Neurosurg 2003:98:959-66.

17 Murayama Y, Vinuela F, Duckwiler GR, et al. Embolization of incidental cerebral aneurysms by using the Guglielmi detachable coil system. J Neurosurg 1999:90:207-14.

18 Lempert TE, Malek AM, Halbach VV, et al. Endovascular treatment of ruptured posterior circulation cerebral aneurysms. Clinical and angiographic outcomes. Stroke 2000;31:100-10.

19 Raymond J, Guilbert F, Weill A, et al. Long-term angiographic recurrences after selective endovascular treatment of aneurysms with detachable coils. Stroke 2003;34:1398-403

20 Gallas S, Pasco A, Cottier JP, et al. A multicenter study of 705 ruptured intracranial aneurysms treated with Guglielmi detachable coils. AJNR Am J Neuroradiol 2005:26:1723-31.

21 Ries T, Siemonsen S, Thomalla G, et al. Long-term follow-up of cerebral aneurysms after endovascular therapy prediction and outcome of retreatment. Am J Neuroradiol 2007;28:1755-61.
22 Renowden SA, Koumellis $\mathrm{P}$, Benes $\mathrm{V}$, et al. Retreatment of previously embolized cerebral aneurysms: the risk of further coil embolization does not negate the advantage of the initial embolization. Am J Neuroradiol 2008;29:1401-4.

23 Campi A, Ramzi N, Molyneux AJ, et al. Retreatment of ruptured cerebral aneurysms in patients randomized by coiling or clipping in the International Subarachnoid Aneurysm Trial (ISAT). Stroke 2007;38:1538-44.

24 Kang HS, Han MH, Kwon BJ, et al. Repeat endovascular treatment in post-embolization recurrent intracranial aneurysms. Neurosurgery 2006;58:60-70.

25 Kawanabe Y, Sadato A, Taki W, et al. Endovascular occlusion of intracranial aneurysms with Guglielmi detachable coils: correlation between coil packing density and coil compaction. Acta Neurochir (Wien) 2001;143:451-5.

26 Sluzewski M, van Rooij WJ, Rinkel GJ, et al. Endovascular treatment of ruptured intracranial aneurysms with detachable coils: long-term clinical and serial angiographic results. Radiology 2003:227:720-4.

27 Ferns SP, Sprengers MES, van Rooij WJ, et al. Coiling of intracranial aneurysms: a systematic review on initial occlusion and reopening and retreatment rates. Stroke 2009;40:e523-9. 